\title{
THE EXISTENCE OF FREE UNIONS IN CLASSES OF ABSTRACT ALGEBRAS
}

\author{
GLORIA C. HEWITT ${ }^{1}$
}

Let $\kappa$ be an ordinal number. A $\kappa$-ary operation on a set $A$ is a mapping $O: A^{\kappa} \rightarrow A$ of all well ordered sequences (of type $\kappa$ ) of elements of $A$ into $A$. Let $\left\langle\kappa_{0}, \cdots, \kappa_{\alpha}, \cdots\right\rangle_{\alpha<\beta}$ be a well ordered sequence of ordinal numbers. An abstract algebra of type $\left\langle\kappa_{0}, \cdots, \kappa_{\alpha}, \cdots\right\rangle_{\alpha<\beta}$ is a system $\left\langle A ; O_{0}, \cdots, O_{\alpha}, \cdots\right\rangle_{\alpha<\beta}$, where $A$ is a nonempty set and $O_{\alpha}$ is a $\kappa_{\alpha}$-ary operation on $A$. The concepts of isomorphism, homomorphism, subalgebra, and direct union of abstract algebras are defined in the usual way (see [2]). Henceforth, we will not distinguish between the abstract algebra $\left\langle A ; O_{0}, \cdots, O_{\alpha}, \cdots\right\rangle_{\alpha<\beta}$ and the set $A$ of its elements. The concept of free union is defined relative to a class $\mathfrak{A}$ of algebras. It will always be assumed that the algebras in $\mathfrak{A}$ are of the same type. Explicit mention of this assumption will usually be omitted.

Definition 1. Let $\mathfrak{A}$ be a class of abstract algebras. Let $\left\{A_{\lambda} \mid \lambda \in \Lambda\right\}$ be a subset of $\mathfrak{A}$. A system $\left\langle A ; h_{\lambda}\right\rangle_{\lambda \in \Lambda}$ consisting of an algebra $A \in \mathfrak{A}$ and a family of projections

$$
h_{\lambda}: A \rightarrow A_{\lambda}
$$

is called a free $\mathfrak{A}$-union of the set $\left\{A_{\lambda} \mid \lambda \in \Lambda\right\}$ if the following extension property is satisfied:

(E) If $\left\{g_{\lambda} \mid \lambda \in \Lambda\right\}$ is a family of homomorphisms $g_{\lambda}: B \rightarrow A_{\lambda}$, where $B \in \mathfrak{A}$, then there is a unique homomorphism $g: B \rightarrow A$ such that $g_{\lambda}=h_{\lambda} \circ g$ for all $\lambda$.

This concept is dual to that of free $\mathfrak{A}$-products studied by D. J. Christensen and R. S. Pierce in [3].

It is not clear that free $\mathfrak{A}$-unions exist. However, if the free $\mathfrak{A}-$ union of a set of algebras does exist, then it is unique in the following sense: for any two free $\mathfrak{A}$-unions

$$
\left\langle A ; h_{\lambda}\right\rangle_{\lambda \in \Lambda} \text { and }\left\langle A ; \bar{h}_{\lambda}\right\rangle_{\lambda \in \Lambda}
$$

of a set $\left\{A_{\lambda} \mid \lambda \in \Lambda\right\} \subseteq \mathfrak{A}$, there exist unique inverse isomorphisms

$$
h: A \rightarrow A \text { and } \bar{h}: \bar{A} \rightarrow A
$$

such that

Presented to the Society, August 31, 1962; received by the editors March 26, 1962.

1 The author is indebted to Professor R. S. Pierce for his valuable suggestions during the preparation of this paper. 


$$
\bar{h}_{\lambda}=h_{\lambda} \circ \bar{h} \text { and } \quad h_{\lambda}=\bar{h}_{\lambda} \circ h \text {. }
$$

The existence and uniqueness of $h$ and $\bar{h}$ come immediately from Definition 1. Since

$$
\bar{h}_{\lambda}=\bar{h}_{\lambda} \circ h \circ \bar{h} \text { and } h_{\lambda}=h_{\lambda} \circ \bar{h} \circ h
$$

$h \circ \bar{h}$ and $\bar{h} \circ h$ are the identity mappings of $\bar{A}$ and $A$ respectively by the uniqueness requirement in $(E)$.

Definition 2. Let $\mathfrak{A}$ be a class of abstract algebras. Let $\left\{A_{\lambda} \mid \lambda \in \Lambda\right\}$ be a subset of $\mathfrak{A}$. Let $\left\langle A ; h_{\lambda}\right\rangle_{\lambda \in \Lambda}$ be a system where $A \in \mathfrak{A}$ and $h_{\lambda}$ is a homomorphism mapping $A$ into $A_{\lambda}$. Define a relation $\sim$ on $A$ as follows: for $x$ and $y$ in $A, x \sim y$ if and only if $h_{\lambda}(x)=h_{\lambda}(y)$ for all $\lambda \in \Lambda$. For $x \in A$, let $\hat{x}=\{y \in A \mid x \sim y\}$. Let $\hat{A}=\{\hat{x} \mid x \in A\}$. Let $\pi$ be the mapping $A \rightarrow \hat{A}$ defined by $\pi(x)=\hat{x}$.

LEMMA 3. (i) $\sim$ is a congruence relation on $A$; that is, an equivalence relation such that

$$
x_{0} \sim y_{0}, \cdots, x_{\mu} \sim y_{\mu}, \cdots, \quad\left(\mu<\kappa_{\alpha}\right)
$$

implies $O_{\alpha}\left\langle x_{0}, \cdots, x_{\mu}, \cdots\right\rangle \sim O_{\alpha}\left\langle y_{0}, \cdots, y_{\mu}, \cdots\right\rangle$.

(ii) $\hat{A}$ is an abstract algebra of type $\left\langle\kappa_{0}, \cdots, \kappa_{\alpha}, \cdots\right\rangle$, with operations $\hat{O}_{\alpha}$ defined by

$$
\hat{O}_{\alpha}\left\langle\hat{x}_{0}, \cdots, \hat{x}_{\mu}, \cdots\right\rangle=\left[O_{\alpha}\left\langle x_{0}, \cdots, x_{\mu}, \cdots\right\rangle\right]^{\wedge} .
$$

Thus the mapping $\pi$ is a homomorphism of $A$ onto $\hat{A}$.

(iii) There is a unique family of homomorphisms $\tau_{\lambda}: \hat{A} \rightarrow A_{\lambda}$ such that $h_{\lambda}=\tau_{\lambda} \circ \pi$. Moreover, the $\tau_{\lambda}$ separate points. If $\hat{A} \in \mathfrak{A}$ and $\left\langle A ; h_{\lambda}\right\rangle_{\lambda \in \Delta}$ is a free $\mathfrak{A}$-union of $\left\{A_{\lambda} \mid \lambda \in \Lambda\right\}$, so is $\left\langle\hat{A}, \tau_{\lambda}\right\rangle_{\lambda \in \Lambda}$.

Proof. The proof of (i) and (ii) is routine.

(iii) Define $\tau_{\lambda}: \hat{A} \rightarrow A_{\lambda}$ by $\tau_{\lambda}(\hat{x})=h_{\lambda}(x) . \tau_{\lambda}$ is clearly well defined and is a homomorphism since $h_{\lambda}$ is. $\hat{x} \neq \hat{y}$ implies there exists $\lambda$ such that $h_{\lambda}(x) \neq h_{\lambda}(y)$. Thus $\tau_{\lambda}(\hat{x}) \neq \tau_{\lambda}(\hat{y})$ and $\left\{\tau_{\lambda} \mid \lambda \in \Lambda\right\}$ separates points. Since $h_{\lambda}$ is onto for all $\lambda, \tau_{\lambda}$ is onto for all $\lambda$ since $h_{\lambda}=\tau_{\lambda} \circ \pi$. To show that $\left\langle\hat{A} ; \tau_{\lambda}\right\rangle_{\lambda \in \Delta}$ is the free $\mathfrak{A}$-union of $\left\{A_{\lambda} \mid \lambda \in \Lambda\right\}$, suppose $g_{\lambda}: B \rightarrow A_{\lambda}$, where $B \in \mathfrak{A}$, is a set of homomorphisms. By (E) of Definition 1, there is a unique homomorphism $j: B \rightarrow A$ such that $g_{\lambda}=h_{\lambda} \circ j$. Define the mapping $g: B \rightarrow A$ by $g=\pi \circ j$. Then

$$
\tau_{\lambda} \circ g=\tau_{\lambda} \circ \pi \circ j=h_{\lambda} \circ j=g_{\lambda} \text {. }
$$

The uniqueness of $g$ follows from the fact that $\left\{\tau_{\lambda} \mid \lambda \in \Lambda\right\}$ separates points.

LEMmA 4. Let $\left\langle A ; h_{\lambda}\right\rangle_{\lambda \in \Lambda}$ be a system having all the properties of 
Definition 1 except possibly the uniqueness of the homomorphism $\mathrm{g}$ in (E). Assume that $\left\{h_{\lambda} \mid \lambda \in \Lambda\right\}$ separates points. Then $g$ is unique.

Conversely, if any homomorphic image of an algebra of $\mathfrak{A}$ is in $\mathfrak{A}$ and $\left\langle A ; h_{\lambda}\right\rangle_{\lambda \in \Lambda}$ is a free $\mathfrak{A}$-union, then $\left\{h_{\lambda} \mid \lambda \in \Lambda\right\}$ separates points.

Proof. Suppose $g$ and $\bar{g}$ are homomorphisms of $B$ such that $h_{\lambda} \circ g=h_{\lambda} \circ \bar{g}$ for all $\lambda \in \Lambda$. Let $b \in B$. Then $h_{\lambda}(g(b))=h_{\lambda}(\bar{g}(b))$ for all $\lambda$ implies $g(b)=\bar{g}(b)$ since $\left\{h_{\lambda} \mid \lambda \in \Lambda\right\}$ separates points.

To prove the converse, notice by Lemma $3,\left\langle\hat{A} ; \tau_{\lambda}\right\rangle_{\lambda \in \Lambda}$ is a free $\mathfrak{A}$-union and $\left\{\tau_{\lambda} \mid \lambda \in \Lambda\right\}$ separates points. Hence there exist unique inverse isomorphisms $i: A \rightarrow \hat{A}$ and $\bar{i}: \hat{A} \rightarrow A$ such that $h_{\lambda}=\tau_{\lambda} \circ i$ and $\tau_{\lambda}=h_{\lambda} \circ \bar{\imath}$ for all $\lambda$. Thus $x \neq y$ implies $i(x) \neq i(y)$ which implies $\tau_{\lambda}(i(x)) \neq \tau_{\lambda}(i(y))$ for some $\lambda$. That is, $h_{\lambda}(x) \neq h_{\lambda}(y)$ for some $\lambda$.

COROLlARY 5. Let $\mathfrak{A}$ be a class of abstract algebras with the property that any homomorphic image of an algebra of $\mathfrak{A}$ is in $\mathfrak{A}$. Let $\left\langle A ; h_{\lambda}\right\rangle_{\lambda \in \Delta}$ be the free union of $\left\{A_{\lambda} \mid \lambda \in \Lambda\right\}$. A ssume $\left\{g_{\lambda} \mid \lambda \in \Lambda\right\}$ is a set of homomorphisms $g_{\lambda}: B \rightarrow A_{\lambda}$, where $B \in \mathfrak{A}$. Then the homomorphism $g: B \rightarrow A$ satisfying $g_{\lambda}=h_{\lambda} \circ g$ for all $\lambda \in \Lambda$ is one-to-one if and only if $\left\{g_{\lambda} \mid \lambda \in \Lambda\right\}$ separates points.

Proof. Suppose $g$ is one-to-one and $x \neq y$ in $B$. Then $g(x) \neq g(y)$. By Lemma 4 , there is a $\lambda$ such that $h_{\lambda}(g(x)) \neq h_{\lambda}(g(y))$.

Conversely, suppose $\left\{g_{\lambda} \mid \lambda \in \Lambda\right\}$ separates points and $x \neq y$ in $B$. Then there is a $\lambda$ such that $g_{\lambda}(x) \neq g_{\lambda}(y)$, or $h_{\lambda}(g(x)) \neq h_{\lambda}(g(y))$. This implies $g(x) \neq g(y)$.

Suppose the class $\mathfrak{A}$ is closed under the formation of direct unions. Let $\left\{A_{\lambda} \mid \lambda \in \Lambda\right\}$ be a subset of $\mathfrak{A}$. Let $A$ be the direct union of $\left\{A_{\lambda} \mid \lambda \in \Lambda\right\}$. Let $h_{\lambda}: A \rightarrow A_{\lambda}$ be the projection homomorphism. That is,

$$
h_{\lambda}\left(\cdots, x^{\lambda}, \cdots\right)=x^{\lambda}, \quad x^{\lambda} \in A_{\lambda} .
$$

Then it is easy to see that $\left\langle A ; h_{\lambda}\right\rangle_{\lambda \in \Lambda}$ is the free $\mathfrak{A}$-union of $\left\{A_{\lambda} \mid \lambda \in \Lambda\right\}$. Thus the existence of direct unions implies the existence of free $\mathfrak{A}$ unions. However, free $\mathfrak{A}$-unions may exist without the existence of direct unions in $\mathfrak{A}$.

We will conclude this paper with an existence theorem for free $\mathfrak{A}$ unions. The following result for free $\mathfrak{A}$-products was proved in [3]: Let $\mathfrak{A}$ be a class of abstract algebras with the following properties:

(i) any algebra isomorphic to an algebra of $\mathfrak{A}$ is in $\mathfrak{A}$;

(ii) any subalgebra of an algebra of $\mathfrak{A}$ is in $\mathfrak{A}$;

(iii) any direct union of algebras of $\mathfrak{A}$ is in $\mathfrak{A}$.

Let $\left\{A_{\lambda} \mid \lambda \in \Lambda\right\}$ be a subset of $\mathfrak{A}$. Suppose that there is an algebra $C$ in $\mathfrak{A}$ and a family of isomorphisms $j_{\lambda}: A_{\lambda} \rightarrow C$. Then the free $\mathfrak{A}$ product of $\left\{A_{\lambda} \mid \lambda \in \Lambda\right\}$ exists. 
It is clear from the proof of this theorem and the comment above that condition (iii) may be replaced by (iii)' any free $\mathfrak{A}$-union of algebras of $\mathfrak{A}$ is in $\mathfrak{A}$. With this small change, we get an existence theorem for free $\mathfrak{A}$-unions which dualizes this result. In the proof of this theorem, we need the following lemma.

Lemma 6. Let $\mathfrak{A}$ be a class of abstract algebras. Let $\left\{A_{\lambda} \mid \lambda \in \Lambda\right\}$ be a subset of $\mathfrak{A}$. Suppose $h_{\lambda}: C \rightarrow A_{\lambda}, C \in \mathfrak{A}$, is a family of mappings which separates points. Let $\beta=\prod_{\lambda \in \Lambda}\left|A_{\lambda}\right|$. Then $|C| \leqq \beta$.

Proof. Let $A$ be the set product of $\left\{A_{\lambda} \mid \lambda \in \Lambda\right\}$. Then $|A|=\beta$. Define $\psi: C \rightarrow A$ by $\psi(x)=\left(\cdots, h_{\lambda}(x), \cdots\right) . \psi$ is one-to-one: $x \neq y$ implies there is a $\lambda$ such that $h_{\lambda}(x) \neq h_{\lambda}(y)$. Thus, $\left(\cdots, h_{\lambda}(x), \cdots\right)$ $\neq\left(\cdots, h_{\lambda}(y), \cdots\right)$ or $\psi(x) \neq \psi(y)$. Hence $|C| \leqq \beta$.

THEOREM 7. Let $\mathfrak{A}$ be a class of abstract algebras with the following properties:

(i) any homomorphic image of an algebra of $\mathfrak{A}$ is in $\mathfrak{A}$;

(ii) any free $\mathfrak{A}$-product of algebras of $\mathfrak{A}$ is in $\mathfrak{A}$.

Let $\left\{A_{\lambda} \mid \lambda \in \Lambda\right\}$ be a subset of $\mathfrak{A}$. Suppose there is an algebra $C \in \mathfrak{A}$ and a family of homomorphisms $h_{\lambda}: C \rightarrow A_{\lambda}$ onto. Then the free $\mathfrak{A}$-union of $\left\{A_{\lambda} \mid \lambda \in \Lambda\right\}$ exists.

Proof. (1) Let $\beta=\prod_{\lambda \in \Lambda}\left|A_{\lambda}\right|$ as in Lemma 6 . Choose a set $M$ of cardinality $\beta$. Let

$$
\mathfrak{B}=\left\{\left\langle B_{\delta} ; g_{\delta \lambda}\right\rangle_{\lambda \in \Lambda} \mid \delta \in \Delta\right\}
$$

where $B_{\delta} \in \mathfrak{A}, B_{\delta}$ is a subset of $M$ and for all $\lambda \in \Lambda, g_{\delta \lambda}$ is a homomorphism mapping $B_{\delta}$ into $A_{\lambda}$. Notice that $\mathfrak{B}$ is a set.

(2) Suppose $h_{\lambda}: B \rightarrow A_{\lambda}, B \in \mathfrak{U}$, is a set of homomorphisms. Then there is a $\delta \in \Delta$ and a homomorphism

$$
g: B \rightarrow B_{\delta}
$$

such that

$$
h_{\lambda}=g_{\delta \lambda} \circ g \quad \text { for all } \lambda \in \Lambda \text {. }
$$

By Lemma $3, \hat{B}$ is an algebra and is the homomorphic image of $B$. So by (i), $\hat{B}$ is in $\mathfrak{A}$. Also by Lemma 3 , there is a set of homomorphisms $g_{\lambda}: \hat{B} \rightarrow A_{\lambda}$ such that $h_{\lambda}=g_{\lambda} \circ \pi$ which separates points. Thus by Lemma $6,|\hat{B}| \leqq \beta$. Hence there is a one-to-one mapping $\gamma: \hat{B} \rightarrow M$. Let $N=\gamma(\hat{B}) \cdot \gamma$ induces operations $O_{0}, \cdots, O_{\alpha}, \cdots,(\alpha<\beta)$, on $N$ so that $\gamma$ becomes an isomorphism. Thus $N$ is an algebra in $\mathfrak{A}$ and is a subset of $M$. Furthermore, $g_{\lambda} \circ \gamma^{-1}$ is a set of homomorphisms from $N$ into $A_{\lambda}$. Hence $\left\langle N ; g_{\lambda} \circ \gamma^{-1}\right\rangle$ is in $\mathfrak{B}$. Let $N=B_{\delta}, g_{\delta \lambda}=g_{\lambda} \circ \gamma^{-1}$ and 
$g=\boldsymbol{\gamma} \circ \pi$ where $\pi$ is the homomorphism of $B$ onto $\hat{B}$. Then $g$ is a homomorphism of $B$ onto $N$ satisfying $g_{\delta \lambda} \circ g=h_{\lambda}$ for all $\lambda \in \Lambda$.

Notice taking $C$ in the last assumption of the theorem to be $B$, we have that $\mathfrak{B}$ is nonempty.

(3) Let $\left\langle D ; i_{\delta}\right\rangle_{\delta \in \Delta}$ be the free $\mathfrak{A}$-product of the algebras of $\mathfrak{B}$. Then there exist homomorphisms

$$
\psi_{\lambda}: D \rightarrow A_{\lambda}
$$

such that

$$
g_{\delta \lambda}=\psi_{\lambda} \circ i_{\delta} .
$$

By (2) and the last assumption of the theorem, there is a $\delta \in \Delta$ and a homomorphism $h: C \rightarrow B_{\delta}$ such that

$$
h_{\lambda}=g_{\delta \lambda} \circ h \quad \text { for all } \lambda \text {. }
$$

This implies $g_{\delta \lambda}$ is onto for all $\lambda$ and hence $\psi_{\lambda}$ is onto for all $\lambda$. Let $\tau_{\lambda}: \hat{D} \rightarrow A_{\lambda}$ be the mappings defined in Lemma 3 . Then $\tau_{\lambda}$ is onto since $\psi_{\lambda}$ is; and $\left\{\tau_{\lambda} \mid \lambda \in \Lambda\right\}$ separates points. It remains to show that $\left\langle\hat{D} ; \tau_{\lambda}\right\rangle$ is the free $\mathfrak{A}$-union of $\left\{A_{\lambda} \mid \lambda \in \Lambda\right\}$.

Suppose $h_{\lambda}: B \rightarrow A_{\lambda}, B \in \mathfrak{A}$, is a set of homomorphisms. By (2), there is a $\delta$ in $\Delta$ and a homomorphism

$$
h: B \rightarrow B_{\delta} \text { satisfying } h_{\lambda}=g_{\delta \lambda} \circ h .
$$

The mapping $\eta=\pi \circ i_{\delta} \circ h$ is a homomorphism from $B$ to $\hat{D}$ and

$$
\tau_{\lambda} \circ \eta=\tau_{\lambda} \circ \pi \circ i_{\delta} \circ h=\psi_{\lambda} \circ i_{\delta} \circ h=g_{\delta \lambda} \circ h=h_{\lambda} .
$$

Since $\left\{\tau_{\lambda} \mid \lambda \in \Lambda\right\}$ separates points, $\eta$ is unique by Lemma 4 .

CoRollary 8. If the class $\mathfrak{A}$ satisfies conditions (i) and (ii) of Theorem 7 , and if in addition, every algebra in $\mathfrak{A}$ contains a one element subalgebra, then every subset of $\mathfrak{A}$ has a free $\mathfrak{A}$-union.

Proof. We need only show that the last condition of Theorem 7 is satisfied. Let $\left\langle C ; i_{\lambda}\right\rangle$ be the free $\mathfrak{A}$-product of $\left\{A_{\lambda} \mid \lambda \in \Lambda\right\}$. For all $\mu \in \Lambda$, let $g_{\lambda \mu}$ be the identity mapping of $A_{\mu}$ if $\lambda=\mu$ and the unique homomorphism of $A_{\lambda}$ onto the one element subalgebra of $A_{\mu}$ if $\lambda \neq \mu$. Then there exist homomorphisms $g_{\mu}: C \rightarrow A_{\mu}, \mu \in \Lambda$, such that

$$
g_{\lambda \mu}=g_{\mu} \circ i_{\lambda} \quad \text { for all } \lambda \in \Lambda \text {. }
$$

Since when $\mu=\lambda, g_{\lambda \mu}$ is onto, $g_{\mu}$ is onto for all $\mu$.

Corollary 9. Let $\mathfrak{A}$ be a class of algebras satisfying (i) and (ii) of Theorem 7. Suppose $\mathfrak{A}$ contains free $\mathfrak{A}$-algebras of arbitrarily large generating sets. Then every subset of $\mathfrak{A}$ has a free $\mathfrak{A}-$ union. 
The free $\mathfrak{A}$-algebra with $\gamma$ generators is an algebra $B \in \mathfrak{A}$ containing a subset $X$ of cardinality $\gamma$ with the property that any mapping of $X$ into an algebra $A \in \mathfrak{A}$ can be uniquely extended to a homomorphism of $B$ into $A$.

Proof of Corollary 9. Let $\left\{A_{\lambda} \mid \lambda \in \Lambda\right\}$ be any subset of $\mathfrak{A}$. Let $\beta=\sup \left\{\left|A_{\lambda}\right| \mid \lambda \in \Lambda\right\}$. Let $F$ be a free algebra in $\mathscr{A}$ whose generating set, $X$, has cardinality greater than or equal to $\beta$. Since $\left|A_{\lambda}\right| \leqq|X|$ for all $\lambda$, there exists a mapping of $X$ onto $A_{\lambda}$ which extends uniquely to a homomorphism of $F$ onto $A_{\lambda}$. Thus the last condition of Theorem 7 is satisfied.

It is proved in [3] that if for the class $\mathfrak{A}$, (i) every algebra isomorphic to an algebra of $\mathfrak{A}$ is in $\mathfrak{A}$; (ii) every subalgebra of an algebra in $\mathfrak{A}$ is in $\mathfrak{A}$; (iii) every free $\mathfrak{A}$-union of algebras in $\mathfrak{A}$ is in $\mathfrak{A}$; (iv) every algebra of $\mathfrak{A}$ contains a one element subalgebra; then every subset of $\mathfrak{A}$ has a free $\mathfrak{A}$-product. This, together with 8 , gives the following theorem.

Theorem 10. If the class $\mathfrak{A}$ satisfies:

(i) any homomorphic image of an algebra in $\mathfrak{A}$ is in $\mathfrak{A}$;

(ii) any subalgebra of an algebra of $\mathfrak{A}$ is in $\mathfrak{A}$;

(iii) every algebra of $\mathfrak{A}$ contains a one element subalgebra; then every subset of $\mathfrak{A}$ has a free $\mathfrak{A}$-product if and only if it has a free $\mathfrak{A}$-union.

\section{REFERENCES}

1. G. Birkhoff, On the structure of abstract algebras, Proc. Cambridge Philos. Soc. 31 (1935), 434-454.

2. , Lattice theory, rev. ed., Amer. Math. Soc. Colloq. Publ. Vol. 25, Amer. Math. Soc., Providence, R. I., 1949.

3. D. J. Christensen and R. S. Pierce, Free products of $\alpha$-distributive boolean algebras, Math. Scand. 7 (1959), 81-105.

Montana State University 